\title{
The formation of the smooth halo component
}

\author{
Jorge Peñarrubia \\ Institute for Astronomy, University of Edinburgh, Royal Observatory, Blackford Hill, \\ Edinburgh EH9 3HJ, UK \\ email: jorpega@roe.ac.uk
}

\begin{abstract}
The detection and characterization of debris in the integral-of-motion space is a promising avenue to uncover the hierarchical formation of the Milky Way. Yet, the fact that the integrals do not remain constant during the assembly process adds considerable complexity to this approach. Indeed, in time-dependent potentials tidal substructures tend to be effaced from the integral-of-motion space through an orbital diffusion process, which naturally leads to the formation of a 'smooth' stellar halo. In this talk I will introduce a new probability theory that describes the evolution of collisionless systems subject to a time-dependent potential. The new theory can be used to reconstruct the hierarchical assembly of our Galaxy through modelling the observed distribution of accreted stars in the integral-of-motion space.
\end{abstract}

Keywords. Galaxy: kinematics and dynamics

\section{Introduction}

Astrometric surveys of our Galaxy offer a unique opportunity to test key aspects of the current cosmological paradigm, wherein galaxies form throug a hierarchical accretion process. In principle, remnants of the Milky Way assembly will appear as stellar clumps in the integral-of-motion space (e.g. energy - angular momentum) that cluster around the integrals of the orbits of the progenitor systems from which they were tidally stripped. Hence, detecting and characterizing individual subtructures can be used to uncover the accretion history of our Galaxy (e.g. Helmi \& White 1999; Helmi \& de Zeeuw 2000). In practice, however, the fact that cosmological potentials evolve with time implies that integrals do not remain constant. Peñarrubia (2013) shows that substructures in the integral-of-motion space undergo collisionless diffusion, and that the inexorable cycle of deposition, and progressive dissolution, of tidal clumps naturally leads to the formation of a 'smooth' stellar halo.

There are several mechanisms that introduce a time dependence in the potential of our Galaxy. For example, cooling and infall of gas deepen the potential well of the host (e.g. adiabatic contraction), whereas supernova feedback may alter the density profile of the dark matter halo (e.g. Ponzten \& Governato 2013). Accretion of massive satellites leads to the growth of the dark matter halo and break existing spatial symmetries, which in turn induces diffusion both in the energy and angular momentum dimensions. In order to uncover the accretion history of the Milky Way from observations of the integral-ofmotion space one needs to model such diffusion processes.

Unfortunately, the relaxation of collisionless systems is still poorly understood. The most successful theory dates back to the violent relaxation theory of Lynden-Bell (1967), which shows that self-gravitating systems evolve toward a unique equilibrium state which can be accurate described by a combination of Fermi-Dirac distribution functions. The central difficulty in accepting the distribution function derived from Lynden-Bell theory is that it predicts infinite mass for the system. In other words, the variational problem that determines the most probable distribution function possesses no solution for any 
finite total mass. This shortcoming may be due to the short life of the process that drives relaxation, i.e. fluctuations of the gravitational field, which vanish on the time scale $(G \rho)^{-1 / 2}$, well before the thermodynamical equilibrium is attained. As a result, in most gravitating systems the evolution will be frozen in a subdomain of the available phase space (a.k.a. 'incomplete relaxation'). In addition, in the current cosmological paradigm galaxies can be rarely found in an equilibrium state. Indeed, the detection of tidal streams in the stellar halo of the Milky Way provides a clear-cut evidence that our Galaxy has not yet reached dynamical equilibrium.

\section{A new probability theory}

Recently, Peñarrubia (2015) proposes a new probability theory that describes the nonequilibrium state of collisionless gravitating systems subject to time-dependent gravitational forces. This theory can be used, for example, to calculate the distribution function of stellar tracers orbiting in a growing potential. In a spherical potential the nonequilibrium distribution function, $f(E, t)=N(E, t) / \omega(E, t)$, where $N$ is the probability to find a particle in the energy interval $E, E+\mathrm{d} E$ at the time $t$ and $\omega$ is the so-called density of states (e.g. Binney \& Tremaine 1986), is found by convolving the initial DF with a transition probability (also called 'propagator') $p_{c}$

$$
N(E, t)=\int \mathrm{d} E_{0} N\left(E_{0}, t_{0}\right) p_{c}\left(E, t \mid E_{0}, t_{0}\right),
$$

where $p_{c}\left(E, t \mid E_{0}, t_{0}\right)$ is the probability that a particle with energy $E_{0}$ at the time $t_{0}$ has an energy $E$ at the time $t$.

Equation (2.1) is a remarkable result, as it suggests that one can describe the dynamical state of a gravitating system at the time $t$ from the state at the time $t_{0}$ through a single probability convolution. This is akin to the "jumps" we are familiar with in Quantum Mechanics! As in Stochastic Calculus, one can also construct a serial sequence of $\kappa$-jumps, $t_{0}<t_{1}<, \ldots,<t_{\kappa-1} \leqslant t_{\kappa}$, with $t_{\kappa}=t_{0}+\tau^{\prime}$, where the end product of a jump is used as the initial condition for the next one. The result is a Markov chain, where

$$
\begin{array}{r}
p_{c, \kappa}\left(E, t \mid E_{0}, t_{0}\right)=\int \mathrm{d} E_{1} p_{c}\left(E_{1}, t_{1} \mid E_{0}, t_{0}\right) \int \mathrm{d} E_{2} p_{c}\left(E_{2}, t_{2} \mid E_{1}, t_{1}\right) \ldots \\
\times \int \mathrm{d} E_{\kappa-1} p_{c}\left(E, t \mid E_{\kappa-1}, t_{\kappa-1}\right) p_{c}\left(E_{\kappa-1}, t_{\kappa-1} \mid E_{\kappa-2}, t_{\kappa-2}\right) .
\end{array}
$$

We shall say that a Markov chain is transitive when the transition probability between the states $t_{0}$ and $t=t_{0}+\tau^{\prime}$ is independent of the number of intermediate steps, that is if $p_{c}=p_{c, 1}=\ldots=p_{c, \kappa}$ for any value of $\kappa$. Peñarrubia (2015) shows that $p_{c}$ is always transitive on time scales $\tau^{\prime} \ll\left(\dot{\Phi} / \Phi_{0}\right)^{-1}$, where $\Phi$ is a time-dependent gravitational potential.

\section{Transition Probabilities}

The probability function $p_{c}\left(E, t \mid E_{0}, t_{0}\right)$ is the result of the convolution of two Gaussians

$$
p_{c}\left(E, t \mid E_{0}, t_{0}\right)=\int p\left(E, t \mid I R^{-2}, t_{0}\right) p\left(I \mid E_{0}, t_{0}\right) \mathrm{d} I .
$$


In potentials that evolve in the linear regime $p$ is the solution to Einstein's equation for freely diffusing particles

$$
p\left(E, t \mid E_{a}, t_{0}\right)=\frac{1}{\sqrt{4 \pi \tilde{D}\left(E_{a}, t\right)}} \exp \left[-\frac{\left[E-E_{a}+\tilde{C}\left(E_{a}, t\right)\right]^{2}}{4 \tilde{D}\left(E_{a}, t\right)}\right],
$$

Here $E_{a}$ denotes the adiabatic energy, which varies with time, and $\tilde{C}$ and $\tilde{D}$ are the so-called drift and diffusion coefficients, respectively. The mean and the dispersion of the distribution are $\overline{E-E_{a}}=-\tilde{C}$, and $\overline{\left(E-E_{a}\right)^{2}}-{\overline{E-E_{a}}}^{2}=2 \tilde{D}$, respectively. If the potential evolves adiabatically both coefficients $\tilde{C}$ and $\tilde{D}$ approach zero, and the probability function (3.2) becomes sharply peaked about $E=E_{a}$, which recovers the adiabatic solution exactly.

The second Gaussian is

$$
p\left(I \mid E_{0}, t_{0}\right)=\frac{1}{\sqrt{4 \pi \tilde{D}\left(E_{0}, t_{0}\right) R_{0}^{4}}} \exp \left[-\frac{\left[I-E_{0} R_{0}^{2}-\tilde{C}\left(E_{0}, t_{0}\right) R_{0}^{2}\right]^{2}}{4 \tilde{D}\left(E_{0}, t_{0}\right) R_{0}^{4}}\right]
$$

where $I=E_{a} R^{2}(t)$ is a dynamical invariant (constant of motion), and $R(t)$ is a scaling factor (see $\S 4$ ).

Hence, to integrate $(2.1)$ and (3.1) we only need to calculate the following functions: $\tilde{C}(E, t), \tilde{C}\left(E_{0}, t_{0}\right), \tilde{D}(E, t)$ and $\tilde{D}\left(E_{0}, t_{0}\right)$. This implies that the problem of collisionless relaxation in the linear regime reduces to the derivation of four coefficients!

\section{Diffusion coefficients}

Peñarrubia $(2013,2015)$ uses dynamical invariants to derive analytically the diffusion coefficients for systems subject to power-law forces

$$
F(r, t)=-\mu(t) r^{n}
$$

In such systems the drift and diffusion coefficients can be simply written as

$$
\tilde{C}(E, t)=-(\dot{R} / R) \dot{\mathcal{I}}
$$

and

$$
\tilde{D}(E, t)=B_{n}(\dot{R} / R)^{2} \mathcal{I} T,
$$

where $B_{n}$ is a positive constant that only depends on the power-law index of the force (4.1). The quantities $\mathcal{I}$ and $T$ are, respectively, the moment of inertia and the mean kinetic energy of a particle ensamble with energy $E$, that is

$$
\begin{aligned}
\mathcal{I}(E, t) & =\left\langle r^{2} / 2\right\rangle=\frac{1}{2 \omega} \int r^{2} \delta(E-H) \mathrm{d}^{3} \mathbf{r d}^{3} \mathbf{v} \\
& =\frac{(4 \pi)^{2}}{2 \omega} \int_{0}^{r_{m}} r^{4}[2(E-\Phi)]^{1 / 2} \mathrm{~d} r
\end{aligned}
$$

and

$$
\begin{aligned}
T(E, t) & =\left\langle v^{2} / 2\right\rangle=\frac{1}{\omega} \int \frac{\mathbf{v}^{2}}{2} \delta(E-H) \mathrm{d}^{3} \mathbf{r d}^{3} \mathbf{v} \\
& =\frac{(4 \pi)^{2}}{6 \omega} \int_{0}^{r_{m}} r^{2}[2(E-\Phi)]^{3 / 2} \mathrm{~d} r
\end{aligned}
$$

where $r_{m}(E, t)$ is the maximum radius that particles with energy $E$ can reach, that is $\Phi\left(r_{m}, t\right)=E$. 
The quantity $R(t)$ is a scaling factor, which for power-law forces is also a power law

$$
R(t)=\left[\frac{\mu(t)}{\mu_{0}}\right]^{-1 /(n+3)} .
$$

Note that $\dot{R} / R=-(\dot{\mu} / \mu) /(n+3)$, and that the diffusion coefficient (4.3) is proportional to the variation of the potential squared. This implies that the diffusion of substructures in the integral-of-motion space is most effective during periods in which the Milky Way potential changes quickly (e.g. during the infall of massive satellites).

\section{N-body tests}

As an illustration, Peñarrubia (2015) considers logarithmic potentials that evolve linearly with time as

$$
\frac{\mu(t)}{\mu_{0}}=1+\epsilon \frac{t-t_{0}}{P_{0}},
$$

where $P_{0}=P\left(E, t_{0}\right)=2 \int_{0}^{r_{m}(E)} \mathrm{d} r / \sqrt{2\left(E-\Phi\left[r, t_{0}\right]\right)}$ is the radial period of an orbit with $r_{m}=\mu_{0}=1$ at $t=t_{0}$.

$N$-body equilibrium realizations of a lowered Maxwellian distribution are generated in a logarithmic potential

$$
f\left(E_{0}, t_{0}\right)= \begin{cases}A\left[e^{\frac{-E_{0}+\Phi_{\lim }}{\sigma^{2}}}-1\right] & , E_{0}<\Phi_{\lim }, \\ 0 & , E_{0} \geqslant \Phi_{\text {lim }},\end{cases}
$$

where $A$ is a normalization factor, and $\Phi_{\text {lim }}=\mu_{0} \ln \left(r_{\text {lim }}\right)$ is an energy truncation. To simplify our models we set $\sigma^{2}=\mu_{0} / 2=3 T$, where $T$ is the kinetic energy associated with the potential (see Table 1).

Next, velocities of all particles are multiplied by a factor $q$. To highlight non-equilibrium features we choose a small value, $q=0.2$, which leads to $|2 T / W|=q^{2}=0.04$. Such a low virial ratio guarantees the collapse of the system on a time-scale comparable to its freefall time. Fig. 1 shows ten snap-shots of a model orbiting in a time-dependent potential that evolves at a rate $\epsilon=-0.1$. Cold collapse happens early on $\left(\tau^{\prime} \approx 0.2 P_{0}\right)$ and leads to the formation of shell structures that move progressively towards larger radii with time. By the end of the simulation, $\tau^{\prime} \approx 2.0 P_{0}$, the model has not yet reached dynamical equilibrium. By definition the system is in a state of 'incomplete relaxation' $\dagger$.

Fig. 2 shows the drift coefficient $\tilde{C}=-(\dot{R} / R)\langle\mathbf{r} \cdot \mathbf{v}\rangle$ as a function of energy for the snapshots shown in Fig. 1 . As the system begins to collapse the averaged radial velocity of the particle ensemble is negative at all radii and $\partial \tilde{C} / \partial t>0$ at all energies. At slightly later times, $\tau^{\prime} \gtrsim 0.2 P_{0}$, the coefficient $\tilde{C}$ begins to exhibit coherent fluctuations in the innermost regions of the potential (left side of the panels), as particles with short orbital period go through pericentre and start moving toward larger radii. In the outskirts, however, particles are still falling in from large distances, which translates into positive values of $\tilde{C}$. The negative crests are associated with the shell features of Fig. 1. Given that in a potential with $n=-1$ the orbital period decreases toward the central regions of the potential as

$$
P(E, t)=(2 \pi / \mu)^{1 / 2} r_{m}=(2 \pi / \mu)^{1 / 2} r_{\lim } \exp (E / \mu),
$$

$\dagger$ These models provide a useful representation of the dynamical state of the outer regions of galactic haloes and galaxy clusters, where dynamical times are comparable to the age of the Universe and phase mixing becomes very inefficient. 
fluctuations in $\tilde{C}$ damp out progressively from inside out. By $\tau^{\prime}=1.9 P_{0}$ the mean radial velocity of particles with $E-\Phi_{\lim } \lesssim-2$ is $\langle\mathbf{r} \cdot \mathbf{v}\rangle \approx 0$, signalling that the inner regions of the system are phase-mixed and evolving in a state of quasi-dynamical equilibrium.

Fig. 3 illustrates the complexity involved in describing the state of systems undergoing violent relaxation. Green dots show the distribution function of the $N$-body models plotted in Fig. 1 at three different snap-shots. The decreasing potential $(\epsilon<0)$ shifts the orbital energies to higher values, which leads to a non-monotonic increase of $f(E, t)$ at fixed energies. Interestingly, the distribution function is not completely smooth. Nonequilibrium features arise in the inner-most regions of the potential and propagate toward high energies as time goes by. After constructing a larger suite of $N$-body models (not shown here) we find that the amplitude of these fluctuations increases for larger $\epsilon$ and smaller $q$ values, which correspond to faster growth rates and higher radial anisotropies, respectively.

Equation (2.1) (red solid lines) is able to capture these complexities and provide an accurate statistical description of the non-equilibrium state of the system. For simplicity we assume that the transition probability $p_{c}\left(E, t \mid E_{0}, t_{0}\right)$ corresponds to a single "jump" between $t_{0}$ and $t_{0}+\tau^{\prime}$. The Green convolution is solved by setting $\tilde{C}\left(E_{0}, t_{0}\right)=0$ and computing $\tilde{D}\left(E_{0}, t_{0}\right)$ analytically from Equation $(4.3)$. The coefficients $\tilde{C}(E, t)$ and $\tilde{D}(E, t)$ are measured from the phase-space coordinates of the $N$-body particles at $t=t_{0}+\tau^{\prime}$ as $\tilde{C}(E, t)=-(\dot{R} / R)\langle\mathbf{r} \cdot \mathbf{v}\rangle$ and $\tilde{D}(E, t)=(\dot{R} / R)^{2}\left\langle(\mathbf{r} \cdot \mathbf{v})^{2}\right\rangle$.

The lower panels of Fig. 3 plots the difference between the $N$-body distribution function and the adiabatic solution at fixed energy values (green dots). From Equation (5.1) particles respond adiabatically to a time-varying force if their orbital periods obey $P\left(\dot{\Phi} / \Phi_{0}\right)=\epsilon\left(P / P_{0}\right) \ll 1$. Since $P$ increases exponentially with the particle energy we find the distribution $N$ evolves adiabatically $\left(\Delta N_{a} \approx 0\right)$ at $E \ll \Phi_{\text {lim }}$, while strong departures from the adiabatic solution $\left(\left|\Delta N_{a}\right| \sim N\right)$ are visible at $E \gtrsim \Phi_{\text {lim }}$, where the potential changes significantly during an orbital period, i.e. $P\left(\dot{\Phi} / \Phi_{0}\right) \sim 1$. As a result, the linear approximation (solid magenta lines) becomes less accurate in the outskirts of the system. Note that similar deviations are also visible in the upper panels at $\tau^{\prime}=1.8 P_{0}$, albeit with a lesser magnitude.

The existence of internal macroscopic motions $(\tilde{C} \neq 0)$ leads to fluctuations of the distribution function which travel toward high energies, as shown in Fig. 2. Comparison with the solid magenta lines shows that the ripples and troughs of the fluctuations are located at energies where the gradient $\nabla(\tilde{C} N) \equiv \partial(\tilde{C} N) / \partial E$ finds local maxima and minima, respectively. Notice that by the end of the simulation $\left(\tau^{\prime}=1.8 P_{0}\right)$ relaxation is still 'incomplete'.

\section{Summary}

Peñarrubia (2015) proposes a probability theory that describes the non-equilibrium evolution of large particle ensembles orbiting in a time-dependent gravitational field. This theory states that in the linear regime the non-equilibrium state of collisionless systems can be obtained by a single convolution of the initial distribution function with a transition probability which is uniquely defined by 4 coefficients, $\tilde{C}(E, t), \tilde{C}\left(E_{0}, t_{0}\right)$, $\tilde{D}(E, t)$ and $\tilde{D}\left(E_{0}, t_{0}\right)$.

In principle these results provide a simple tool to determine the evolution of stellar tracers in time-dependent potentials. In particular, it offers a simple method for modelling the evolution of accreted clumps in the integral-of-motion space of the Milky Way as follows: given the observed energy distribution function of the $i$-th clump, say $N_{i}(E, t)$, we would like to constrain the initial energy distribution $N_{i}\left(E_{\mathrm{acc}}, t_{\mathrm{acc}}\right)$, where $t_{\mathrm{acc}}$ is 


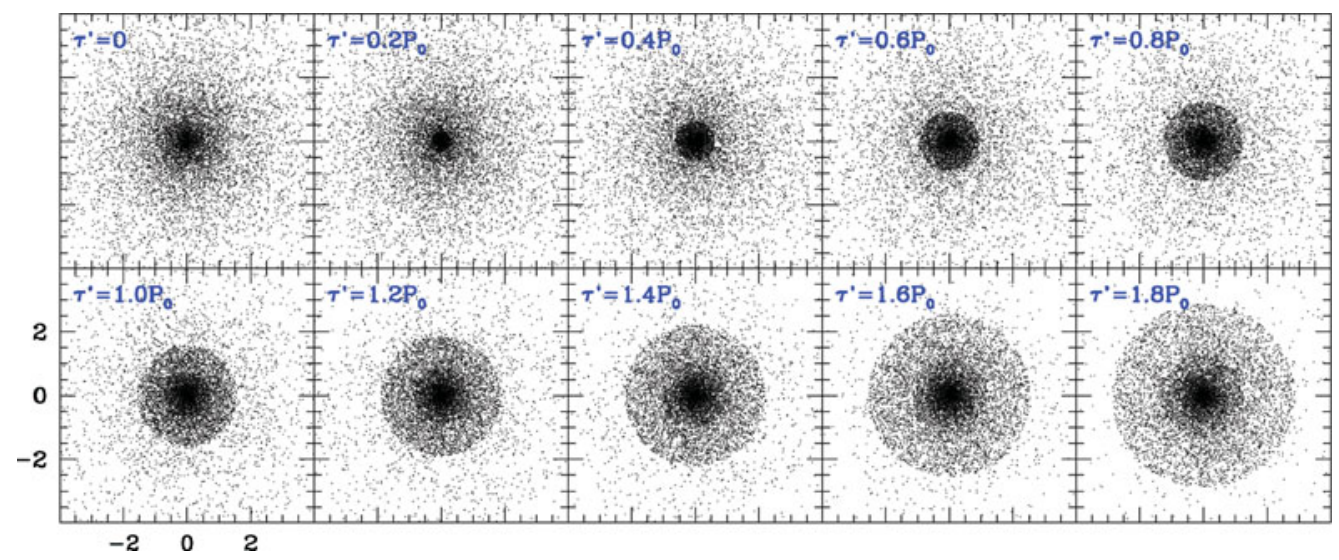

Figure 1. Cold collapse of a model with $|2 T / W|=0.04$ at $t=t_{0}$. Particles move in a time-dependent potential that varies at a constant rate $\epsilon=-0.1$. The system as a whole evolves toward an equilibrium configuration following a 'violent relaxation' process.

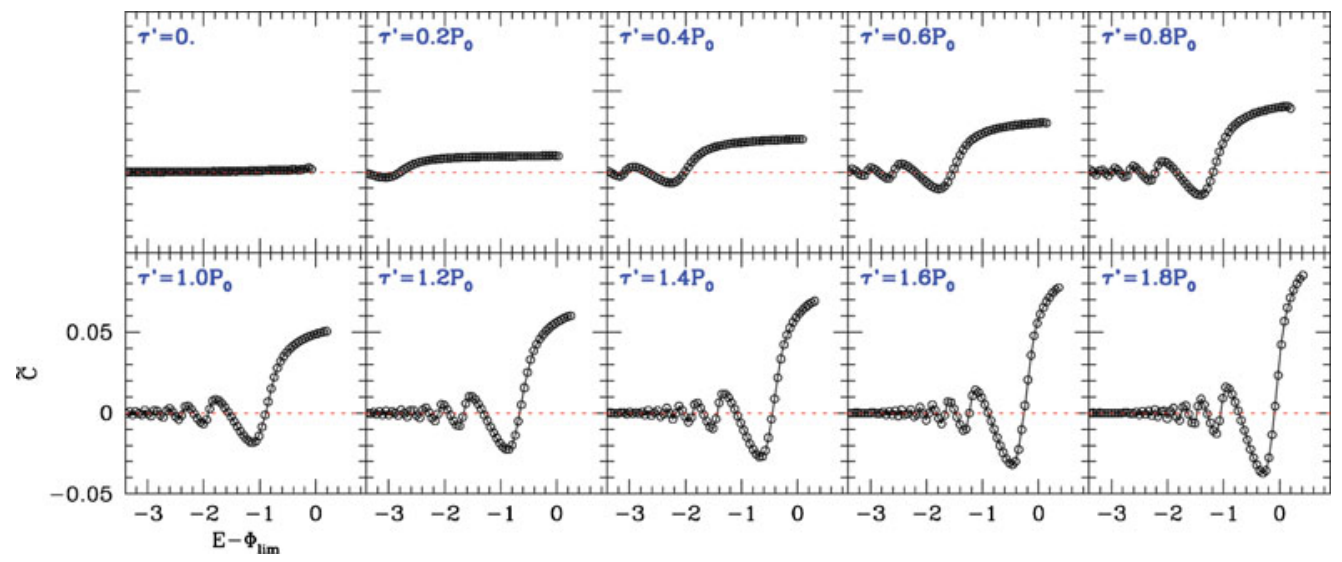

Figure 2. Drift coefficient $\tilde{C}=-(\dot{R} / R)\langle\mathbf{r} \cdot \mathbf{v}\rangle$ as a function of energy of the model shown in Fig. 1. Notice that at $t=t_{0}$ the system is phased mixed (i.e. $\langle\mathbf{r} \cdot \mathbf{v}\rangle=0$ ) but out of virial equilibrium, $|2 T / W|=0.04$. By the end of the simulation only the internal regions of the potential have reached a state of quasi-dynamical equilibrium $(\tilde{C} \approx 0)$.

the time of accretion, for a given potential evolution $\Phi(r, t)$. Both $N_{i}\left(E_{\text {acc }}, t_{\text {acc }}\right)$ and $\Phi(r, t)$ are unknowns and need to be modelled. The former contains information on the properties of the $i$-th progenitor system, e.g. accretion time, orbit, mass, etc. The latter informs us how the Milky Way potential has changed from $t=t_{\text {acc }}$ to the present and has the same functional form for all clumps detected in the integral-of-motion space.

In practice not all potentials admit analytical solutions to the diffusion equation. For example, in systems that are not initially virialized a derivation of the diffusion coefficients requires precise knowledge of the trajectories of individual particles in phase space, rendering the problem analytically intractable. In those cases the diffusion coefficients and the integrals (2.1) and (3.1) need to be solved numerically.

As a final remark it is worth mentioning that the new probability theory has further applications than those mentioned here. For example, we may get a deep insight into the evolution and stability of self-gravitating collisionless systems by studying the evolution 


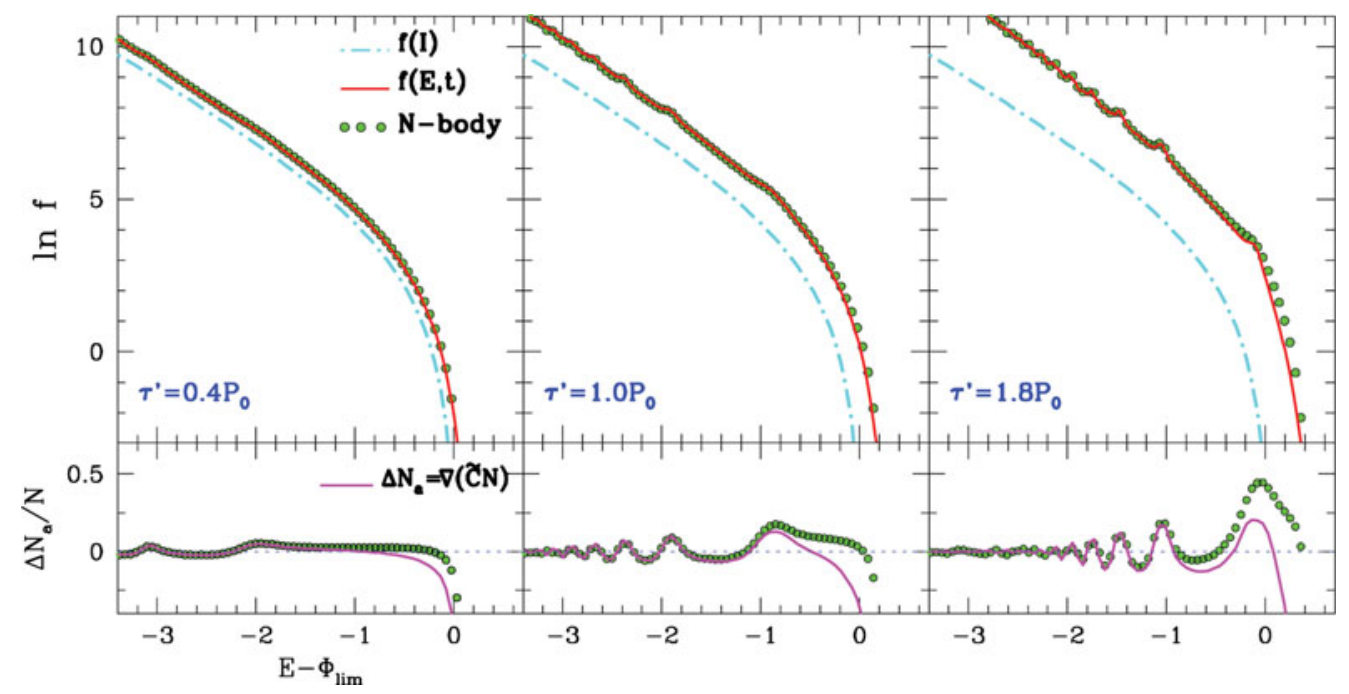

Figure 3. Upper panels: Distribution function $f=N / \omega$ of the models shown in Fig. 1 at three different snap-shots. Red solid lines correspond to the Green convolution given by Equation (2.1) with coefficients $\tilde{C}(E, t)=-(\dot{R} / R)\langle\mathbf{r} \cdot \mathbf{v}\rangle$ and $\tilde{D}(E, t)=(\dot{R} / R)^{2}\left\langle(\mathbf{r} \cdot \mathbf{v})^{2}\right\rangle$ measured from the phase-space locations of the $N$-body particles. Lower panels: Deviation of the $N$-body models from the adiabatic distribution (green dots). Magenta solid lines show deviations from the adiabatic distribution function calculated with our probability theory. Note that the ripples and troughs of the curves are located at energies where the gradient $\nabla(\tilde{C} N)$ finds local maxima and minima, respectively. Departures from the adiabatic solution are particularly strong in the outskirts of the system, $E \gtrsim \Phi_{\lim }$.

of particle ensembles driven by their own self-gravity, i.e.

$$
\nabla^{2} \Phi(\mathbf{r}, t)=4 \pi G \int f(\mathbf{r}, \mathbf{v}, t) \mathrm{d}^{3} v .
$$

Also, our formalism can be straightforwardly extended to systems with a varying spatial symmetry, where both the angular momentum and the energy of individual particles are allowed to vary with time. In this case the Green function that solves the diffusion equation has now one extra dimension, i.e. $p\left(E, L, t \mid E^{\prime}, L^{\prime}, t_{0}\right)$, which defines the probability that a particle with an energy $E^{\prime}$ and angular momentum $L^{\prime}$ at the time $t=t_{0}$ has an energy in the interval $E, E+\mathrm{d} E$ and an angular momentum in the interval $L, L+\mathrm{d} L$ at the time $t=t_{0}+\tau^{\prime}$. Finally, the fact that the evolution of the microcanonical distribution has the same form as Einstein's equation for purely stochastic processes offers the tantalizing possibility to incorporate the effects of random particle-particle collisions into our probability theory in a natural way. Such an extension may be useful, for example, to study a range of dynamical processes taking place in planetary systems, dense stellar objects and/or self-interacting dark matter (SIDM) haloes.

\section{References}

Helmi, A. \& de Zeeuw, P. T. 2000, MNRAS, 319, 657

Helmi, A. \& White, S. D. M. 1999, MNRAS, 307, 495

Lynden-Bell, D. 1967, MNRAS, 136, 101

Peñarrubia, J. 2015, MNRAS, 451, 3537

Peñarrubia, J. 2013, MNRAS, 433, 2576

Pontzen, A. \& Governato, F. 2013, MNRAS, 430, 121 